\title{
Evaluating Abnormal Condition in Physiological Disorder Using a Fluctuation Characteristic of Plant Bioelectric Potential
}

\author{
Shin-ichi Shibata*, Fumitake Sato ${ }^{1}$, Haruhiko Kimura ${ }^{2}$ and Takashi Oyabu ${ }^{3}$ \\ School of Informatics, Department of Information System, Daido University, \\ 10-3 Takiharu, Minami, Nagoya, Aichi 457-8530, Japan \\ 'JSS Co., Ltd., 2-115 Shimeno, Kanazawa, Ishikawa 920-0058, Japan \\ ${ }^{2}$ Graduate School of Natural Science \& Technology, Kanazawa University, \\ Kakuma, Kanazawa, Ishikawa 920-1192, Japan \\ ${ }^{3}$ Kokusai Business Gakuin College, 6-12 Minami, Kanazawa, Ishikawa 920-0919, Japan
}

(Received January 15, 2014; accepted June 3, 2014)

Key words: plant bioelectric potential, evaluating growth condition, maximum entropy method, fluctuation characteristics, logistic regression analysis

For maintaining a steady food supply, the trend has been toward developing factorylike production methods in Japan. To date, there has been no indicator that can predict the growth status of crops. We have accordingly focused on the bioelectric potential inside the plant, which could be an indicator of plant growth. However, a few research studies have been conducted on its role in determining the plant growth status. In this study, we aimed to use plant bioelectric potential as a means of determining growth conditions. Fluctuation characteristics in plant bioelectric potential frequency components should provide indicators of conditions between normal and inhibited growth processes. We discuss that different growth condition factors were identified using logistic regression analysis. This experiment yields a discrimination rate of $86.6 \%$ obtained using the calculated logistic regression model between these conditions.

\section{Introduction}

Recently, both the supply and safety of food have become increasing concerns, owing to environmental issues.(1) At the same time, the decline in the agricultural population and the low food self-sufficiency ratio are also becoming an issue in Japan. According to estimates by the Ministry of Agriculture and Fisheries in 2010, Japan's self-sufficiency rate was $39 \%$ by its calories and the elderly make up $60 \%$ of the agricultural population. ${ }^{(1,2)}$ The productivity per unit area in Japan is high compared with those in other countries,

"Corresponding author: e-mail: shibatas@daido-it.ac.jp 
owing to the skill of seasoned farmers. ${ }^{(3)}$ However, the impending retirement of these skilled farmers, approximately $62 \%$ of whom are over 65 years old, is a serious concern. Therefore, to maintain and improve self-sufficiency, the productivity of young and inexperienced farmers needs to increase.

The difference between skilled and inexperienced farmers is the ability to make considerable judgments. ${ }^{(4)}$ Skilled farmers, on the basis of their experience, can respond to the growth status of their crops. For example, they can diagnose plant diseases by visual inspection and determine the appropriate treatment. Making a sound decision about plant growth conditions requires much background knowledge regarding plants. Nowadays, evaluating the freshness and quality of crops has to depend on inspection by skilled practitioners, which necessitates determining optimal growth conditions by objective evaluation. If standardized production can be conducted by objective evaluation, farmers can steadily supply food owing to the solution of these issues. In this study, we discuss the application of plant information to the objective evaluation of plant growth conditions.

There are several methods suitable for measuring parameters necessary for growth, including nitrogen level and dryness. ${ }^{(4)}$ However, these methods are invasive because they require collecting plant parts for analysis; therefore, it is difficult to use these methods in growing crops. For this reason, noninvasive means of evaluating growth such as chlorophyll fluorescence or 3D diagnostic imaging have become part of agricultural research. ${ }^{(5)}$ However, all these methods are useful only after the conditions deteriorate. They cannot be used to make predictions before the conditions deteriorate. The use of bioelectric potential as a noninvasive means of evaluating growth is being investigated. The plant bioelectric potential is the potential difference caused by variations in the concentrations of ions in the plant cell membranes. It has been shown to vary according to environmental conditions and the growth process. ${ }^{(6-9)}$ However, little research has been conducted on the bioelectric potential as an indicator of crop growth. ${ }^{(10,11)}$ If the bioelectric potential inside the plant could provide direct information about the physiology and surrounding environment, this information could be applied to effectively manage the growth environment.

We have been studying the use of plant bioelectric potential as a means of determining the growth state, using the already established chlorophyll fluorescence as a standard. Previously, we tested the suitability of bioelectric potential measurements under conditions that would definitely damage plant growth. The results showed that there was a difference between the frequency components of the bioelectric potential before and after spraying plants with herbicide, indicating physiological disorder. ${ }^{(12)}$

In this study, we evaluated bioelectric potential frequency components using chlorophyll fluorescence as a standard. A discriminatory model was formulated on the basis of the results of the logistic regression analysis of frequency components calculated from the characteristics of the fluctuation in the bioelectric potential as compared with an abnormal condition. A system of diagnosing growth based on the bioelectric potential inside the plant may aid in determining the growth state before this becomes visually apparent on the surface of the plant. 


\section{Experimental Methods}

\subsection{Experimental plant}

The easily cultivated ornamental plant pothos (Epipremnum aureum) was used in this study. It grows well over a wide range of temperatures, has broad leaves, and is amenable to experimentation. Figure 1 shows a photograph of this plant. Six subject plants were used in the experiment and controlled in the same environment. After initial measurements, herbicide was sprayed on each of the plants, and analysis was performed on the plants with significantly different chlorophyll fluorescence values.

\subsection{Experimental system}

A simplified diagram of the plant bioelectric potential measuring equipment is shown in Fig. 2. The recording electrode came from an electroencephalogram (EEG) (NE117A, NIHONKODEN). The electrode was attached to the leaf surface of the plant using an electrically conducting paste (Z-401CE, NIHONKODEN). The ground was placed in the soil in the plant pot. Because the plant bioelectric potential is very small, the potential from the electrode was amplified by a differential amplifier (MEG-2100, NIHONKODEN) through an input box (JB-220J, NIHONKODEN). A filter circuit was incorporated into the amplifier to remove noise. To remove noise from the utility power, a band-pass filter $(0.08-300 \mathrm{~Hz})$ and a HUM filter were used. The amplified potential was converted to a digital signal using an $\mathrm{A} / \mathrm{D}$ converter and interfaced to a $\mathrm{PC}$, which analyzed the data. The sampling frequency for the bioelectric potential was $100 \mathrm{~Hz}$. The measurement time was $1 \mathrm{~min}$ and each plant was measured 16 times. The measurement period was 2 weeks. Because the bioelectric potential was very small, the effect of noise was a concern. All measuring equipment and plants were accordingly placed in a shield box. We blocked out light in the experiment room and experimented under fluorescent light to remove sunshine effects on measurement time. The illuminance was approximately 340 lux in the measurement time range considered.
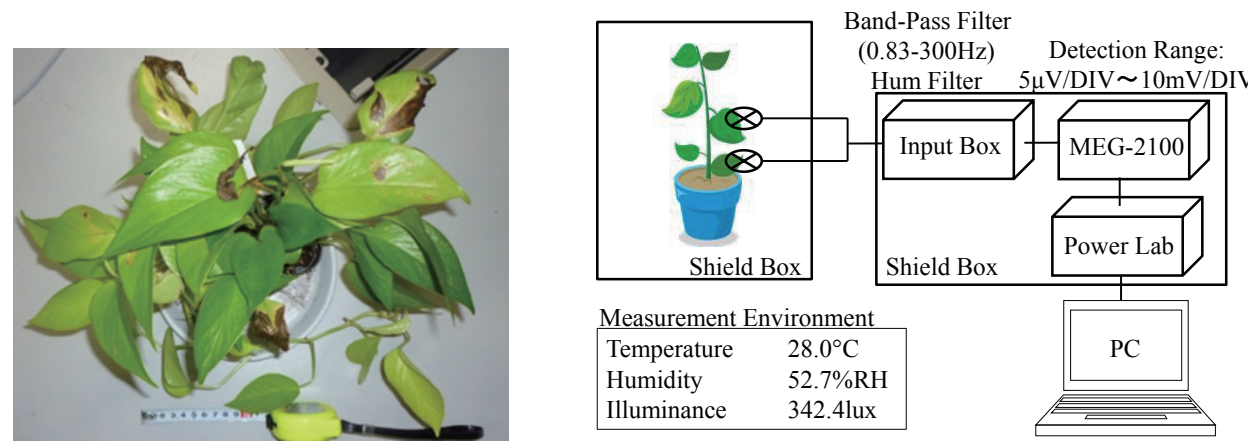

Fig. 1 (left). (Color online) Photograph of subject plant.

Fig. 2 (right). (Color online) Measurement system in this experiment. 


\subsection{Experimental agricultural chemical herbicides}

The herbicide used in the experiment was Nekosogi Quick Pro (Rainbow Pharmaceutical Co., Ltd., registration number 21593). The liquid herbicide (15 ml) was diluted 100 times, in accordance with the specifications. The amount of herbicide used was based on the volume of the pot, which was calculated from the internal diameter of the pot $(9 \mathrm{~cm}$; pot 3$)$. The herbicide $(1.17 \mathrm{ml})$ was sprayed twice onto the surfaces of the leaves on days 1 and 9 of measurement.

\section{Methods of Analysis}

\subsection{Chlorophyll fluorescence}

Growth inhibition should become apparent 3-4 d after the herbicide application. This is the time required for the suppression of growth factors in the plant particularly vital amino acids and proteins. Chlorophyll fluorescence is used as a noninvasive means of evaluating the photosynthesis. The chlorophyll fluorescence value is expressed as the plant growth condition and the value of approximately 0.7 indicates that the plant is healthy. This response was taken as an indicator for evaluating growth. ${ }^{(13)}$ In this experiment, measurements were made before and for 1 week after herbicide application. The fluctuations in electrical potential were evaluated with reference to changes in the chlorophyll fluorescence value. The chlorophyll fluorescence was measured using a pulse-modulator-type chlorophyll fluorescence meter (MINI-PAM, WALZ). A photograph of this measurement procedure is shown in Fig. 3. Chlorophyll $\mathrm{Y}\left(F_{\mathrm{v}} / F_{\mathrm{m}}\right)$ was measured on the surfaces of the leaves to which the plate electrodes for electric potential measurement were attached. $F$ refers to the fluorescence intensity (photon emission reaction rate). $F_{\mathrm{v}} / F_{\mathrm{m}}$ is an index of chlorophyll fluorescence based on a typical saturation pulse measurement. It represents the largest quantum yield for the photosynthesis II chemical pathway, as calculated using eq. (1).

$$
F_{\mathrm{v}} / F_{\mathrm{m}}=\frac{\left(F_{\mathrm{m}}-F_{0}\right)}{F_{\mathrm{m}}}
$$

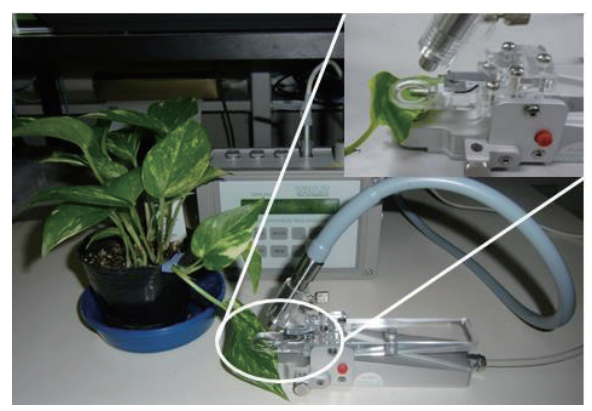

Fig. 3. (Color online) Photograph of measurement procedure using chlorophyll fluorescence measured by MINI-PAM. 
Here, $F_{0}$ is the instantaneous minimum fluorescence yield captured between small fluctuations. $F_{\mathrm{m}}$ is the maximum fluorescence yield. $F_{\mathrm{v}}$ is calculated from the difference between $F_{0}$ and $F_{\mathrm{m}}$. Because of variations in the measurements, six replicates were recorded.

\subsection{Characteristics of fluctuation in plant bioelectric potential}

In this section, we describe the analysis of data collected according to the method explained in $\S 2.2$. Time series data for bioelectric potentials can vary considerably owing to artificial stimuli such as the amount of light or direct contact. ${ }^{(7,10-12)}$ The tiny and irregular wave forms from internal sources are accordingly difficult to detect.

Prior attempts to find a correlation between the plant bioelectric potential and the plant physiological response have been made by exposing the plant to different environmental pollutants. ${ }^{(14-16)}$ These studies showed a correlation between pollutant gases and bioelectric potential. After the injection of pollutants, the power spectrum showed a reduction. In this present study, we also focused on frequency component analysis as a means of measuring correlations particularly the use of the characteristics of the fluctuation in frequency component as a discrimination model. The fluctuation analysis method is shown in Fig. 4. First, the bioelectric potential signal frequencies are determined, and a straight line is approximated using both logarithmic axes. The characteristic values are used as a means of formulating a model that can identify the slope and tangent of the approximate straight line calculated. In the frequency analysis, we did not use conventional fast Fourier transformation (FFT), instead, we used the maximum entropy method (MEM). ${ }^{(17)}$ MEM for limited data provides a spectral estimate by maximizing the autocorrelation function entropy. This method was used by Spcana, which is the software for frequency analysis ${ }^{(18)}$ in this study because it is suitable for time series data over a short time frame and for measurement with high noise levels.
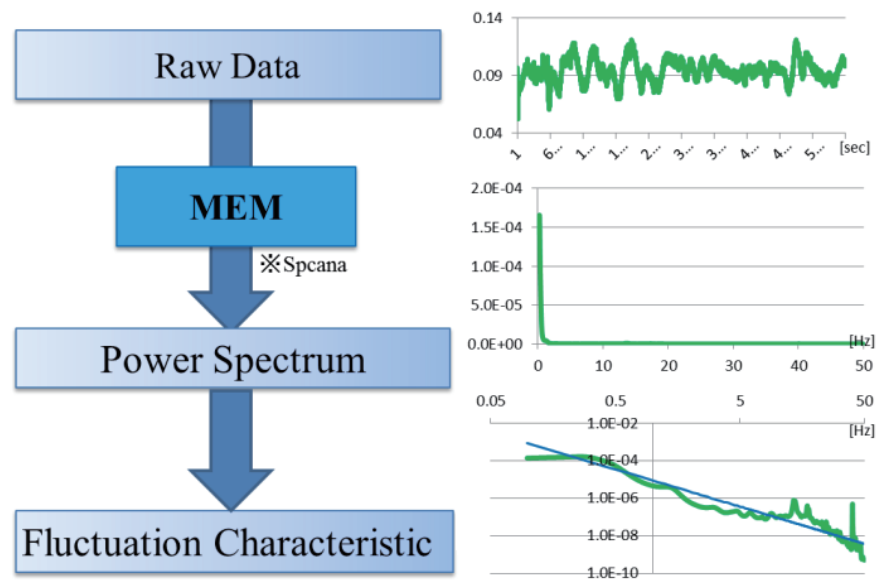

Fig. 4. (Color online) Analysis approach for fluctuation characteristics. 


\subsection{Logistic regression}

Logistic regression is a method of analysis that models an explanatory sigmoid curve using explanatory variables to explain objective variables. The objective variables are represented by categorical variables. It is one method of multivariate analysis used to construct a model for the presence and absence of certain events, such as disease outbreaks, car accidents, or earthquakes. ${ }^{(19,20)}$ Logistic regression analysis was chosen for its special features. The explanatory variable need not follow a normal distribution, and the method also allows the effect of the objective variable on each explanatory variable to be determined. These features make it useful for identifying different parameters. The state of the plant was fitted as an objective variable (see below), on the basis of the slope and intercept of the explanatory variable.

- 0: Growth inhibited

- 1: Growth normal

The growth conditions were characterized using individual differences in chlorophyll fluorescence on the basis of a model derived from logistic regression analysis to generate new test data.

\section{Experimental Result}

\subsection{Chlorophyll fluorescence}

Figure 5 shows individual differences in chlorophyll fluorescence produced by the herbicide, and the data represents the mean and standard deviation of chlorophyll fluorescence. The first data set was recorded before herbicide application, and the eighth was recorded 1 week after herbicide application. The ninth set of measurements was made before the second herbicide application, and the sixteenth was recorded 1 week after the second application. There were two herbicide applications, and the data provide a comparison between 1 week before and after. For two of the six plants, the chlorophyll fluorescence after the second herbicide application fell to less than 0.4, and there was a significant difference between the ninth and sixteenth data points. ${ }^{(1)}$ The chlorophyll fluorescence value decreased markedly on the sixteenth. The surface color of leaves on the sixteenth turned from green to pale green or yellow. In contrast, there was no

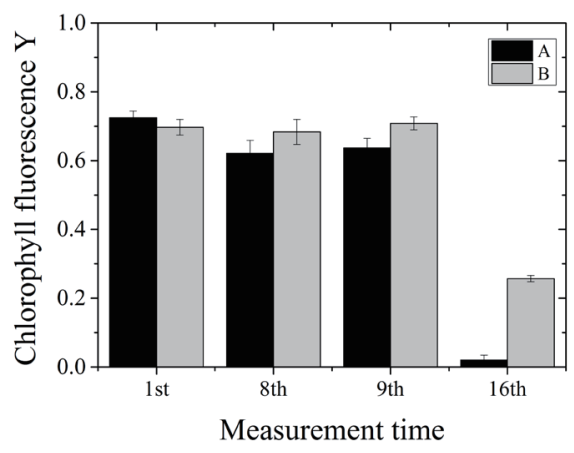

Fig. 5. Chlorophyll fluorescence values of subjects A and B as a function of measurement time. 
significant difference observed among the other four subject plants. The characteristics of the fluctuation in the bioelectric potential in two plants, A and B, were evaluated.

\subsection{Plant bioelectric potential}

The fluctuation characteristics in the two plants that showed a significant difference in chlorophyll fluorescence were calculated for each $4 \mathrm{~d}$ period. The distribution of these fluctuation characteristics is shown in Fig. 6. The vertical axis shows the slope and the horizontal axis shows the tangent in these characteristics. The data groups for the fluctuation characteristics on day 16 (marked with a $\boldsymbol{\nabla}$ ) tend to be grouped at the top left, compared with the points for the other 3 days (1st marked with a square, 8th marked with a circle, and 9th marked with a triangle). The data for day 16 show the effect of growth inhibition, which caused the chlorophyll fluorescence to drop below the standard value of 0.4 . In this study, we assumed that the data for $3 \mathrm{~d}(1 \mathrm{st}, 8 \mathrm{th}$, and 9 th) is the growth normal condition under which the values are above 0.6 , and that the data for day 16 is the growth inhibited condition because of the half value of approximately 0.7 , which is observed under the healthy condition.

Figure 7 shows the approximate curves generated from the fluctuation characteristics over $4 \mathrm{~d}$. The approximate curves from chlorophyll fluorescence values of 0.4 or less (dashed line) are different from the curves for which the chlorophyll fluorescence value exceeded 0.6.

The above results show that the sixteenth data set represents growth inhibition, whereas the sets for the other $3 \mathrm{~d}$ show normal growth. Linear discrimination is achieved by logistic regression analysis.

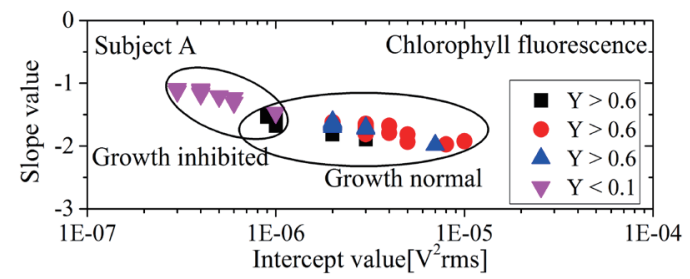

(a)

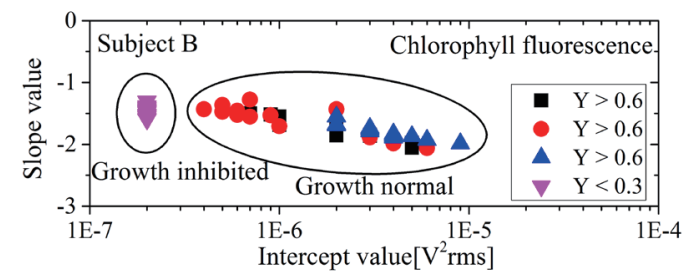

(b)

Fig. 6. (Color online) Scatter plots in fluctuation characteristics [(a) subject A and (b) subject B].

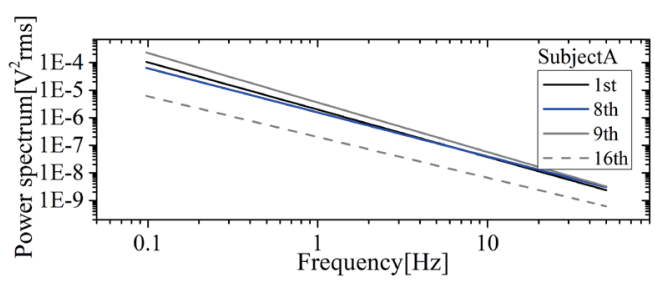

(a)

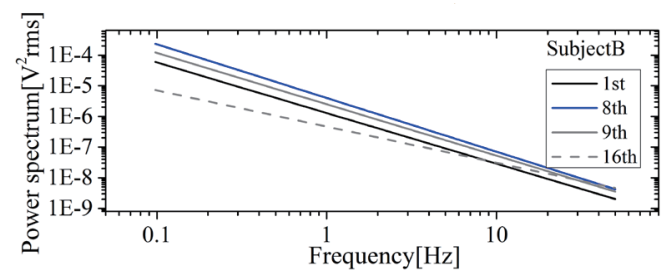

(b)

Fig. 7. (Color online) Approximate curve in fluctuation characteristics [(a) subject A and (b) subject B; both logarithmic axes]. 


\subsection{Logistic regression analysis}

The fluctuation characteristics of the two plants (A and B) described in the previous section were subjected to logistic regression analysis by first analyzing the data. Three explanatory variables were compared: the slope, the intercept, and both variables. The results are shown in Tables $1-3$. This table shows that the discrimination rate was higher when both variables, rather than the fluctuation characteristics, were used. The calculated logistic regression equation is shown below as

$$
\log \frac{p}{1-p}=1.130 x_{\text {intercept }}-12.877 x_{\text {slop }}+53.297 \text {. }
$$

Next, the test data were identified using eq. (2), and the growth status of the test data (C, D, $\mathrm{E}$, and F) was determined. The test data came from the test plants measured on the other days, where there were significant differences between chlorophyll fluorescence values. The three explanatory variables were compared and the results are shown in Table 4.

Table 4 shows that even for the test data, using both fluctuation variables as a discriminant increased the discrimination rate. Furthermore, for the test plant D1, the discrimination rate was low, regardless of the feature used. Table 5 shows the standard

Table 1

Result of discrimination rate in the case of using slope.

\begin{tabular}{llccc}
\hline & & \multicolumn{2}{c}{ Predicted condition } & \multirow{2}{*}{ Discrimination rate } \\
\cline { 2 - 5 } & & 0: Growth inhibited & 1: Growth normal & \\
\hline \multirow{2}{*}{ Correct condition } & 0: Growth inhibited & 20 & 10 & $66.7 \%$ \\
\cline { 2 - 5 } & 1 Growth normal & 4 & 87 & $95.6 \%$ \\
\hline Percentage total & & & $88.4 \%$ \\
\hline
\end{tabular}

Table 2

Result of discrimination rate in the case of using the intercept.

\begin{tabular}{llrrr}
\hline & & \multicolumn{2}{c}{ Predicted condition } & Discrimination rate \\
\cline { 2 - 5 } Correct condition & 0: Growth inhibited & 1: Growth normal & \\
\cline { 2 - 5 } & 1: Growth normal & 27 & 3 & $90.0 \%$ \\
\hline Percentage total & & 4 & 87 & $97.8 \%$ \\
\hline
\end{tabular}

Table 3

Result of discriminination rate in the case of using the intercept.

\begin{tabular}{llrrr}
\hline & & \multicolumn{2}{c}{ Predicted condition } & \multirow{2}{*}{ Discrimination rate } \\
\cline { 2 - 5 } Correct condition & 0: Growth inhibited & 1: Growth normal & \\
\cline { 2 - 5 } & 1: Growth inhibited & 29 & 1 & $96.7 \%$ \\
\hline Percentage total & & 2 & 89 & $97.6 \%$ \\
\hline
\end{tabular}


Table 4

Results of discrimination rate in relation to test data set.

\begin{tabular}{|c|c|c|c|c|c|c|c|c|c|c|}
\hline & & $\mathrm{C} 1$ & $\mathrm{C} 2$ & D1 & D2 & E1 & E2 & F1 & F2 & Average \\
\hline Chlorophyll & orescence Y & 0.65 & 0 & 0.66 & 0.4 & 0.54 & 0.42 & 0.64 & 0.49 & \\
\hline \multirow{3}{*}{$\begin{array}{l}\text { Indification } \\
\text { percentage }\end{array}$} & Slope & $100.0 \%$ & $100.0 \%$ & $12.5 \%$ & $100.0 \%$ & $100.0 \%$ & $100.0 \%$ & $75.0 \%$ & $100.0 \%$ & $85.9 \%$ \\
\hline & Intercept & $100.0 \%$ & $60.0 \%$ & $50.0 \%$ & $100.0 \%$ & $100.0 \%$ & $100.0 \%$ & $68.8 \%$ & $100.0 \%$ & $84.8 \%$ \\
\hline & $\begin{array}{c}\text { Slope and } \\
\text { intercept }\end{array}$ & $100.0 \%$ & $80.0 \%$ & $12.5 \%$ & $100.0 \%$ & $100.0 \%$ & $100.0 \%$ & $100.0 \%$ & $100.0 \%$ & $86.9 \%$ \\
\hline
\end{tabular}

Table 5

Standard deviation among fluctuation characteristics in test data set.

\begin{tabular}{lcc}
\hline Standard deviation & 0: Growth inhibited & 1: Growth normal \\
\hline Slope & 0.34 & 0.17 \\
\hline Intercept & 3.91 & 2.56
\end{tabular}

deviations for the fluctuation characteristics calculated from the bioelectric potentials. The standard deviations for both the slope and the intercept were 1.5 times greater in the healthy plants than in the unhealthy plants.

\section{Discussion}

The experimental results show that the tangent and slope of the fluctuation characteristics for plants in a normal state of growth approximate a normal distribution. The tangent and slope are reduced in the growth-inhibited plants. The herbicide creates unfavorable growth conditions by inhibiting photosynthesis. During photosynthesis, the chlorophyll in chloroplasts extracts an electron from water with the addition of light. Blocking this function appears to be reflected in a reduction in bioelectric potential, reducing the power spectrum and tangent. Random features similar to white noise are said to become strong as the slope of the fluctuation shows a decline. ${ }^{(21)}$ The results appear to show that the growth state of the plant affects the fluctuation characteristics of the bioelectric potential, regardless of individual differences.

The logistic regression analysis shows that using the slope and tangent as explanatory variables provides the best means of discrimination, although the accuracy of discrimination is low for plant D1. A possible explanation is that the standard deviations of the fluctuation characteristics are larger for the normal growth state than for the growth-inhibited state. The chlorophyll fluorescence for plant D1 was 0.66 , the highest value among all the test data. However, these experiments have shown that if the chlorophyll fluorescence value is 0.4 or lower, it may be possible to identify the growth state of the plant. 


\section{Conclusions}

In this paper, we describe experiments on measuring the bioelectric potential of plants with inhibited growth as a way of determining their growth state and finding a means of evaluating plant growth. We formulated a model for discriminating the growth state using the characteristics of the fluctuation in the bioelectric potential of growthinhibited plants to improve our knowledge of how to evaluate plant growth. In this study, six plants were sprayed with a herbicide, and those that showed a difference in chlorophyll fluorescence were subjected to logistic regression analysis to identify a discriminant function. We used a herbicide that inhibited photosynthesis as a standard way of determining the growth state. Photosynthetic inhibition can be measured by chlorophyll fluorescence. Plants that showed a significant difference in chlorophyll fluorescence were subjected to the frequency analysis of their bioelectric potential using MEM. Each coefficient in the fluctuation characteristics was treated as a feature to devise a discrimination model.

Logistic regression analysis showed that discrimination power was higher when both the slope and intercept features were used together than when either was used alone. As a supplementary experiment, when the plants with a significant difference in chlorophyll fluorescence were tested, using the already calculated discriminatory model, a discrimination rate of $86.6 \%$ was obtained. When the individual chlorophyll fluorescence values dropped to 0.4 or below, the growth state could be predicted to some extent.

In this study, we demonstrated that it is possible to distinguish two distinct growth states: healthy and unhealthy. However, it is necessary to evaluate gradations in plant health in more detail and to determine the length of time that growth inhibition has been occurring. Additional experiments on finer gradations of plant growth state using a larger number of plants are required.

\section{References}

1 M. Takatsuji: J. Soc. High Technol. Agric. 22 (2010) 2 (in Japanese).

2 M. Takatsuji: A Plant Factory (Koudansha Ltd., Tokyo, 1979) pp. 5-7 (in Japanese).

3 A. Shinjo: IPS 51 (2010) 635 (in Japanese).

4 M. Takatsuji: A Completely Controlled Plant Factory (Ohmsha Ltd., Tokyo, 2007) Chap. 2 (in Japanese).

5 X. Jin, S. Oshita, Y. Makino and Y. Kawagoe: J. Soc. High Technol. Agric. 25 (2013) 102 (in Japanese).

6 Y. Miwa: The Japan Society of Mechanical Engineers. 90 (1987) 76 (in Japanese).

7 T. Shimbo, M. Fujii, A. Sawada, T. Oyabu and H. Kimura: IEEJ Trans. SM. 127 (2007) 425 (in Japanese).

8 K. Ando, Y. Hasegawa, H. Maekawa and T. Katsube: IEICE Trans. Electron. 91 (2008) 1905.

9 H. Kobayashi, S. Kobayashi, K. Yamada and T. Yachi: IEICE Technical Report. EE. 106 (2006) 53 (in Japanese).

10 M. Kurita and K. Nakabayashi: J. Soc. High Technol. Agric. 19 (2007) 117 (in Japanese).

11 K. Ando, Y. Hasegawa, T. Yaji, and H. Uchida: IEEJ Trans. SM. 132 (2012) 159 (in Japanese). 
12 S. Shibata, F. Satou, H. Kimura and T. Oyabu: IEEJ Trans. SM. 132 (2012) 154 (in Japanese).

13 A. Tanaka, H. Usuki and F. Kenji: Tree Health. 13 (2009) 12 (in Japanese).

14 Y. Hasegawa, S. Asada, T. Katsube and T. Ikeguchi: IEICE Trans. Electron. E87-C (2004) 2093.

15 T. Oyabu, A. Sawada, K. Takenaka and B. Wolverton: Sens. Actuators 89 (2003) 131.

16 S. Shibata, S. Lixin, H. Kimura and T. Oyabu: EICA. 15 (2011) 65 (in Japanese).

17 T. Kojima, N. Hiroshi, Y. Yoshizaki and H. Soeda: Japanese Society of Fisheries Engineering. 2 (2001) 145 (in Japanese).

18 M. Ozaki, T. Ohishi, T. Mori and Y. Kayaba: Technical Note of PWRI 4248 (2012) 59 (in Japanese).

19 K. Onishi, D. Ichikawa and H. Harada: Japanese Society of Stomato-Pharyngology. 19 (2006) 38 (in Japanese).

20 Raghavendra B.K and S.K Srivatsa: IJCSS 5 (2011) 503.

21 M. Hirose and M. Adachi: IEICE Technical Report. NLP. 109 (2009) 209 (in Japanese). 